\title{
APROXIMAÇÃO DO CORPO UNIFORME PARA PORTA-AMOSTRAS DE DIMENSÕES REDUZIDAS NA ANÁLISE TÉRMICA DIFERENCIAL
}

\author{
JOÃO BAPTISTA DOMICIANO \\ KLEMENSAS R. JURAITIS ${ }^{1}$ \\ AIRTON STORI ${ }^{2}$
}

DOMICIANO, J.B.; JURAITIS, K.R.; STORI, A. Aproximação do corpo uniforme para porta-amostras de dimensões reduzidas na análise térmica diferencial. Semina: Ci. Exatas/TecnoL, Londrina, v. 13,

n. 4, p.231-237, dez. 1992.

RESUMO: Muitos métodos podem ser utilizados para o estudo das propriedades térmicas de materiais através da técnica de Análise Térmica Diferencial (ATD). O método experimental usual de obtenção da curva de ATD exige a construção de portaamostras com geometria previamente planejada para facilitar o tratamento teórico da transmissão do calor, o que muitas vezes não é um problema simples. Neste trabalho, descrevemos a aplicabilidade do Método da Aproximação do Corpo Uniforme para porta-amostras de dimensões reduzidas, na ATD. Uma das vantagens deste método consiste em poder considerar o aquecimento ou esfriamento do sistema, constituído pela amostra e porta-amostras, como um aquecimento ou esfriamento Newtoniano. Isto simplifica o tratamento teórico.

PALAVRAS-CHAVES: Análise Térmica Diferencial; Transmissão de Calor

\section{1 - INTRODUÇÃO}

Quando um campo térmico dá origem a gradientes de temperatura no interior de um material, sabe-se que ocorre transferência de calor por condução. Se considerarmos uma lâmina de um certo material, com secção reta de área $A$, espessura $\mathrm{Dx}$ e com as faces mantidas a temperaturas diferentes, uma quantidade de calor $D Q$ se transmite perpendicularmente às faces durante 0 intervalo de tempo Dt. A experiência mostra que $D Q$ é diretamente proporcionai a Dt e $A$, para uma dada diferença de temperatura DT; também mostra que, se DT e Dx forem pequenos, $D G$ será diretamente proporcional a DT/Dx para Dt e $A$ constantes. Portanto,

$$
\frac{\Delta Q}{\Delta t} \propto A \frac{\Delta T}{\Delta x}
$$

No limite, se a lâmina tiver espessura infinitesimal $d x$, através da qual existe uma diferença de temperatura $d T$, temos a lei fundamental da condução de caior

$$
\frac{d Q}{d t}=-\mathcal{K} \cdot \mathcal{A} \cdot \frac{d T}{d x}
$$

Onde $d Q / d t$ é a taxa de transferência de calor, através da área $A ; d T / d x$ é o gradiente de temperatura e $K$ é uma constante de proporcionalidade chamada condutividade térmica, que é uma propriedade do material condutor do calor.

O conhecimento da taxa $d Q / d t$ de transferência de calor é de fundamentai importância em muitas técnicas de análises dos materiais, como na Análise Térmica Diferencial (ATD), de nosso interesse, neste artigo.

O método experimental usual de obtenção da curva ATD consiste no aquecimento (ou esfriamento) da amostra e do material de referência em igualdade de condições, fazendo-se o registro simultâneo da temperatura da amostra e da diferença de temperaturas entre a amostra e o material de referência (BROWN, 1988). O método usual é este porque utiliza um processo de transmissão de calor a uma taxa constante no tempo, o que simplifica a análise teórica.

Os equipamentos usuais que possibilitam a utilização do processo de transmissão de calor numa taxa constante tem porta-amostras do tipo mostrado na Figura 1, os quais, salvo ligeiras variações, consistem de um bloco cilíndrico, geralmente de cobre ou alumínio, com duas cavidades maiores S1 E S2, respectivamente para a amostra e a material de referência (WENDLANDT, 1986). As cavidades $S 1$ e $S 2$ devem ser de tamanhos e formas rigorosamente iguais. Termopares são ligados de maneira adequada em S1 e S2 para fornecer as temperaturas da amostra e do material de referência. O tratamento do problema envolve, portanto, a propagação do

1 - Departamento de Física da Universidade Estadual de Londrina, Caixa Postal 6001, CEP 86051-970, Londrina, Pr., Brasil

2 - Pós-Graduado "Lato-sensu" - Curso de Especialização em Física da Universidade Estadual de Londrina 
calor, por condução; a simetria do problema é simplificada porque a construção das cavidades é feita de modo a tirar proveito da simetria cilíndrica.

O equipamento de ATD montado no Laboratório de Materiais e Instrumentação do Departamento de Física, cuja calibração foi trabalho de Monografia do Curso de Especialização em Física (STORI, 1990), opera com o método do fluxo de calor constante. Contudo não só o porta-amostras é diferente dos usuais, como também é inovadora a técnica utilizada para se conseguir o aquecimento (ou esfriamento) da amostra com taxa constante.

Em r osso equipamento, conforme descrito adiante, o fornecimento de calor à amostra ou a retirada de calor da mesma é feito através do fluxo de ar, com o auxílio de um compressor. O fluxo de ar é ajustável por um rotâmetro. No aquecimento o ar passa por um resistor elétrico que o aquece; chega ao porta-amostras, aquecendo-o. e, consequentemente, aquece a amostra, conforme a Figura 2. No aquecimento, a superfície cilíndrica externa do porta-amostras é a primeira a receber o fluxo de ar aquecido; assim, o calor se propaga de fora para dentro do porta-amostras cilíndrico, por condução, aquecendo a amostra, também cilíndrica, que se encontra no seu interior. A medida que aumenta a temperatura na superfície externa do porta-amostras, aumenta também a temperatura da amostra. No esfriamento a superfície cilíndrica externa do porta-amostras é a primeira a perder calor para o fluxo de ar, mais frio; assim, o calor propaga-se do interir da amostra para fora do porta-amostras cilíndrico, por condução, esfriando a amostra. Assim, num tratamento rigoroso do problema, deveríamos considerar uma propagação de calor, por condução, no estado estacionário, uma vez que ambas as temperaturas variam da mesma maneira com o tempo. No que se segue, mostraremos como este tratamento pode ser simplificado.
Suporte de Isolante

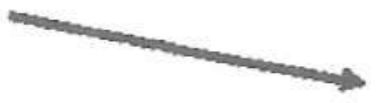

Tubos Condutores

para os

Termopares
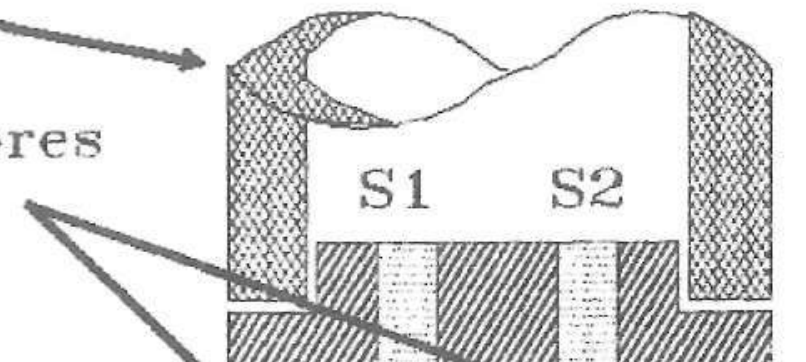

(1) -
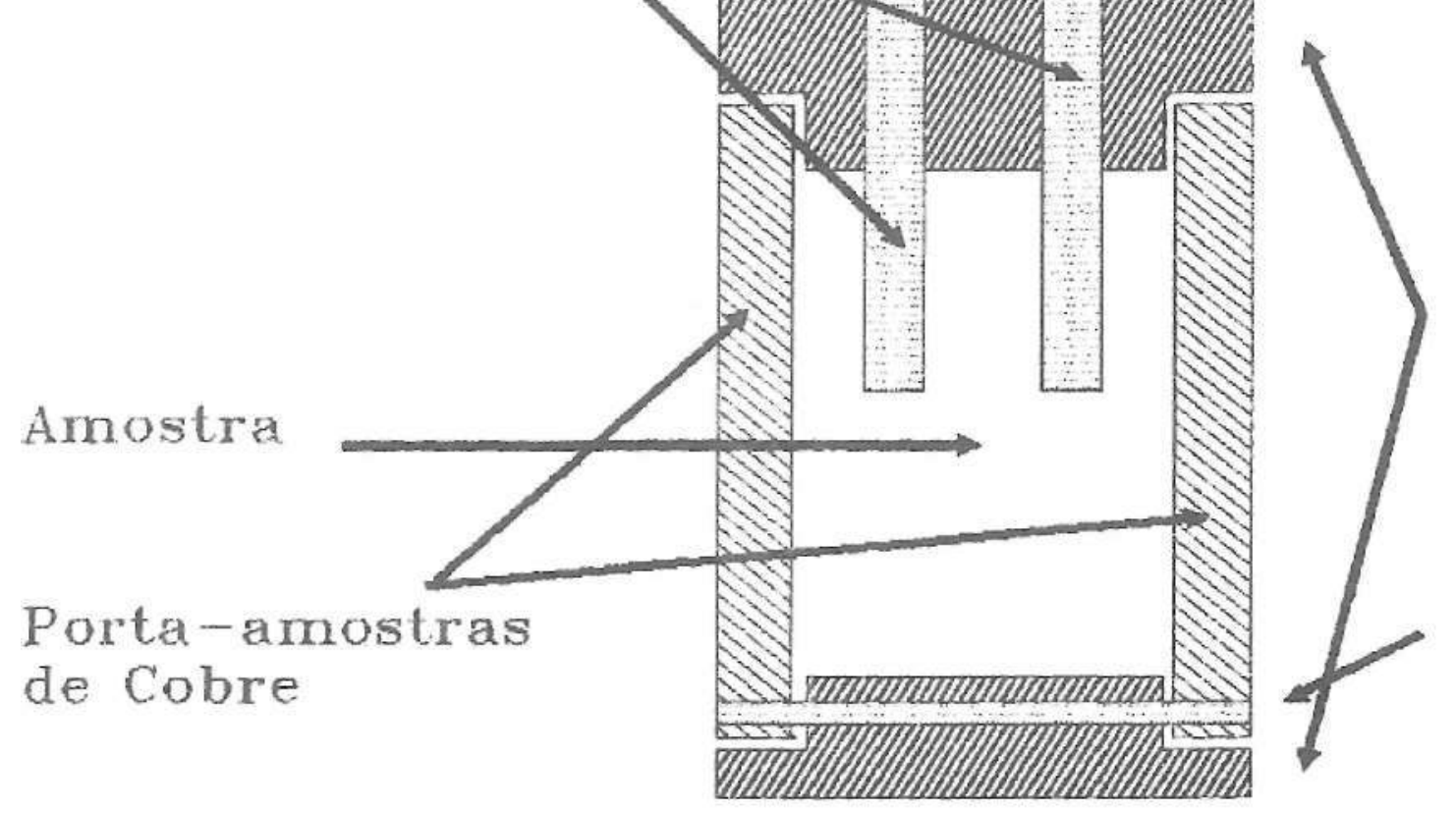

Juntas

Isoladoras

DETALHE DO
SISTEMA
PORTA-AMOSTRA

Cavilha

FIGURA 1 - Desenho esquemático do porta-amostras utilizado nas nossas experiências 


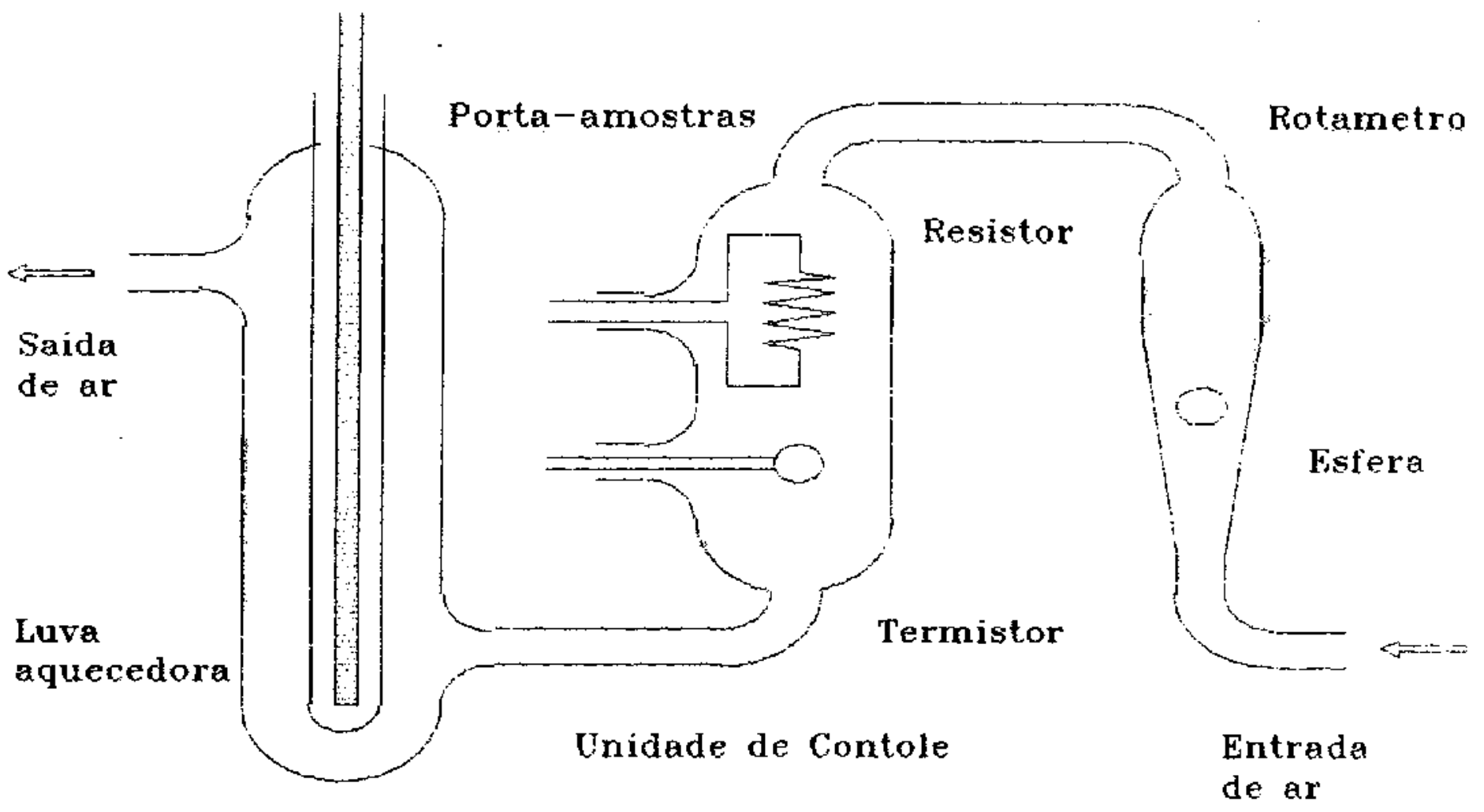

SISTEMA DE CONTROLE DE TEMPERATURAS

FIGURA 2 - Desenho esquemático do sistema de cantrole de temperaturas, mostrando o respectivo fluxo de ar que controla a transferéncia de calor para o portanamostras

\section{2- APROXIMAÇÃO DO CORPO UNIFORME}

De acordo com S1SS0M \& PITTS (1979), o tratamento para qualquer problema de transferência de calor em regime transiente deveria começar com a necessidade de considerar o conhecimento dos gradientes de temperaturas internos. Entretanto, segundo o mesmo autor, para sistemas suficientemente pequenos, como é o caso do sistema amostra mais porta-amostra do nosso equipamento, o problema da condução de calor pode ser razoavelmente aproximado pela hipótese da temperatura uniforme, e a análise envolve o uso de uma capacidade térmica ligada. Em nosso caso, significa que o sistema amostra mais porta-amostra pode ser considerado como um corpo uniforme imerso no fluxo de ar, onde tanto a amostra quanto a superfície externa, que é o nosso material de referência, são aquecidos simultaneamente, em idênticas condições. Nosso sistema porta-amostras, conforme veremos, foi construído com dimensões reduzidas visando atender à aproximação do corpo uniforme. $O$ aquecimento ou esfriamento do mesmo comporta-se como um aquecimento ou esfriamento Newtoniano. Por isso, antes de discutirmos a aproximação do corpo uniforme, analisaremos a lei de esfriamento de Newton.

A lei de esfriamento, de Newton, trata da taxa de esfriamento de um corpo com temperatura $T$, quando 0 mesmo é colocado num ambiente, normalmente o ar, em equilíbrio térmico em certa temperatura $T_{a}<T$, sendo que a diferença $\left(T-T_{a}\right)$ não ultrapasse uma ou duas dezenas de graus Celsius. O corpo perde calor para o ambiente numa taxa dG/dt. Segundo a lei do esfriamento, de Newton:

$$
\frac{d Q}{d t}=C \cdot A \cdot\left(T-T_{\text {it }}\right)
$$

Onde $C$ é denominada coeficiente de transferência de calor e $A$ é a área do corpo exposta ao ambiente. Visto que no esfriamento

$$
\frac{d Q}{d t}<0 \text { e } T-T_{a}>0, \text { então } \mathcal{C}<0
$$

Lembrando da primeira lei da termodinâmica, ou seja, 


$$
d l^{-}=d Q-d I^{-}
$$

e que, neste caso $d W=0$, pois não há trabalho realizado sobre o corpo, temos $d U=d Q$. Então $d O=m_{L} C_{p} d T$ e podemos reescrever a expressão (3):

$$
\begin{aligned}
\frac{d T}{d t} & =\frac{\mathcal{C} \cdot \mathcal{A}}{m \cdot(T)}\left(T-T_{t}\right) \\
& =-K^{*} \cdot\left(T-T_{a}\right)
\end{aligned}
$$

Admitindo que o ambiente praticamente não varia de temperatura com a presença do corpo aquecido, a temperatura final deste ê $T_{f}=T_{a}$, e a equaçăo (4) fica:

$$
\frac{d T}{\left(T-T_{f}\right)}=-K^{*} . d t
$$

Integrando desde $\circ$ instante inicial, $\left(t_{i}=0 ; T_{i}\right)$, até um instante genérico $\{t ; T\}$, e considerando $K$ constante com a temperatura, o que é válido na faixa de trabałho de nosso equipamento, teremos:

$$
\left.\ln \left|T-T_{f}\right|\right|_{l^{\prime}=T_{i}} ^{T}=-k^{*} . t
$$

\section{Curvas Típicas Newtonianas}

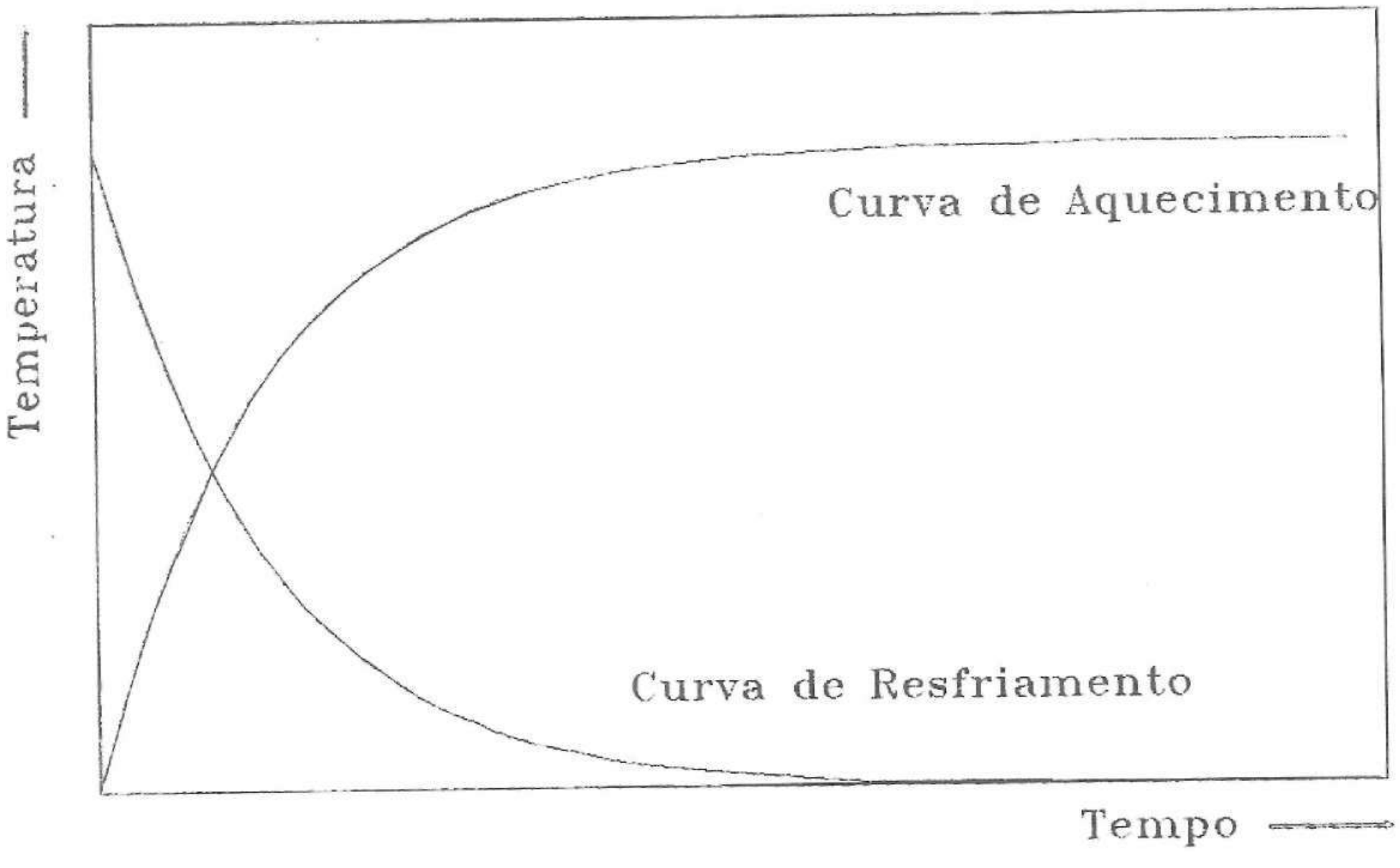

FIGURA 3 - Registro da variação da temperatura em função do tempo do tipo Newtoniano. (a) Resfriamento, (b) Aquecimento. 
Voltando à Aproximação de Corpo Uniforme, lembremos que o sistema porta-amostras do nosso equipamento constitui-se de uma casca cilindrica de cobre, de dimensōes muito pequenas $(L=10 \mathrm{~mm}$ e $R=3 \mathrm{~mm}$ ), com bases superior e inferior de material isolante térmico. A casca cilíndrica é preenchida de amostra e o conjunto permanece numa corrente de ar, com temperatura que pode variar de maneira controlada.

Podemos denominar $T_{\infty}$ a temperatura da corrente de ar, visto que esta ế uma fonte inesgotável de calor. Suponhamos que o cilindro esteja inicialmente aquecido, tal que $T_{\infty}<T_{C}$, sendo $T_{C}$ a temperatura do eixo interior do cilindro. Conforme já descrito, no esfriamento da amostra, o movimento do ar sobre o porta-amostras resulta numa transferência de calor convectiva entre a superflcie externa do cilindro porta-amostras e a corrente de ar; tal transferência de calor pode ser expressa pela lei de Newton, do esfriamento:

$$
\frac{\overline{d Q}}{d t}=\bar{h} \cdot \mathcal{A}_{s} \cdot\left(T_{s}-T_{\infty}\right)
$$

onde $\bar{h}$ é um coeficiente médio de transferência de calor, e o subscrito (s) refere-se à superfície do cilindro. No esfriamento esta energia deve conduzir-se da superfície para a corrente e, na superficie, é igual à energia proveniente do interior da amostra, transferida por condução, ou seja, pela equação (2) da condução de çalor:

$$
\frac{d Q}{d t}=-\left.\mathcal{K} \cdot \mathcal{A}_{s} \cdot \frac{d T}{d r}\right|_{r=R}
$$

Assim, podemos igualar as equações (8) e (9) pois elas representam quantidades iguais, que são a taxa de calor que chega à superfície externa do porta-amostras, por condução, e a taxa de salda de calor da superfície externa do porta-amostras para a corrente de ar:

$$
\begin{aligned}
\bar{h} \cdot\left(T_{s}^{\prime}-T_{\infty}\right) & =-\left.\mathcal{K} \cdot \frac{d T}{d r}\right|_{r=R} \\
& \approx-\mathcal{K} \cdot \frac{T_{c}-T_{s}}{R}
\end{aligned}
$$

A aproximação ê tanto mais verdadeira quanto menor for o raio $R$ do cilindro. Reagrupando-se os termos, vem:

$$
\frac{T_{c}-T_{s}}{T_{s}-T_{\infty}}=\frac{\bar{h} . R}{\mathcal{K}} \equiv \text { número de Biot }
$$

Vê-se que o número de Biot pode ser interpretado como a razão entre a resistência do fluxo de calor interno e a resistência do fluxo de calor externo. Assim, a aproximação da temperatura uniforme é tanto melhor quanto menor for o número de Biot, pois isto indica que o corpo possui resistência interna desprezível ao fluxo de calor, e podemos fazer uma análise considerando-se a capacidade térmica da ligação. Então, para sistemas com número de Biot suficientemente pequeno, o problema da condução de calor pode ser razoavelmente aproximado pela hipótese de temperatura uniforme, e a análise teórica envolve o uso de uma capacidade térmica ligada.

A aproximação da temperatura uniforme ao sistema amostra mais porta-amostra do nosso aparelho é válida visto que $R$ é pequeno, a casca cilíndrica é muito fina e o material tem elevada condutividade térmica $K$. Cálculos aproximados indicam um módulo de Biot da ordem de $10^{-3}$ para o sistema porta-amostras do nosso arranjo experimental. A primeira lei da Termodinâmica aplicada ao sistema conduz ao balanço, em módulo:

Fluxo de calor = Variação da energia durante $\mathrm{dt}$ térmica durante $\mathrm{dt}$

Logo,

$$
\bar{h} \cdot \mathcal{A}_{s} \cdot\left(T-T_{\infty}\right) \cdot d t=C_{t} \cdot d T
$$

Onde $C_{t}$ es uma capacidade térmica associada ao sistema. No esfriamento temos $\left(T-T_{\infty}\right)>0$ e $d T<0$ e, portanto:

$$
\frac{\bar{h} \cdot \mathcal{A}_{s}}{C_{t}} \cdot d t=-\frac{d T}{T-T_{\infty}}
$$

cuja solução exige o conhecimento da condição inicial $T=T_{i}$ para $t_{i}=0$, ficando:

$$
\left.\ln \left(\left|T-T_{\infty}\right|\right)\right|_{T=T_{i}} ^{T}=-\frac{\bar{h} \cdot \mathcal{A}_{s}}{C_{t}} \cdot t
$$

No esfriamento temos:

$\ln \left(T-T_{\infty}\right)-\ln \left(T_{i}-T_{\infty}\right)=-\frac{\bar{h} \cdot \mathcal{A}_{s}}{C_{t}} . t$ ou seja,

$$
\frac{T-T_{\infty}}{T_{i}-T_{\infty}}=e^{-\frac{\bar{h} \cdot A_{s}}{r_{t}} \cdot t}
$$

e a históría da temperatura corresponde a um decaimento exponencial análogo ao decaimento da voltagem num capacitor elétrico. Por analogia, podemos escrever:

$$
\frac{T-T_{\infty}}{T_{i}-T_{\infty}}=e^{-\frac{1}{\mathcal{R C}}}
$$

onde $\mathrm{RC}_{\mathrm{t}}$ é a constante de tempo térmica do sistema. Podemos, ainda, reescrever, após rearranjo:

$$
T=T_{i}+\left(T_{\infty}-T_{i}\right) \cdot\left(1-e^{-\frac{t}{\mathcal{R} C_{t}}}\right)
$$

que $\measuredangle$ idêntica à equação (7).

Semina, Ci. Exatas/Tecnol., v. 13, n. 4, p. 231-237, dez. 1992 


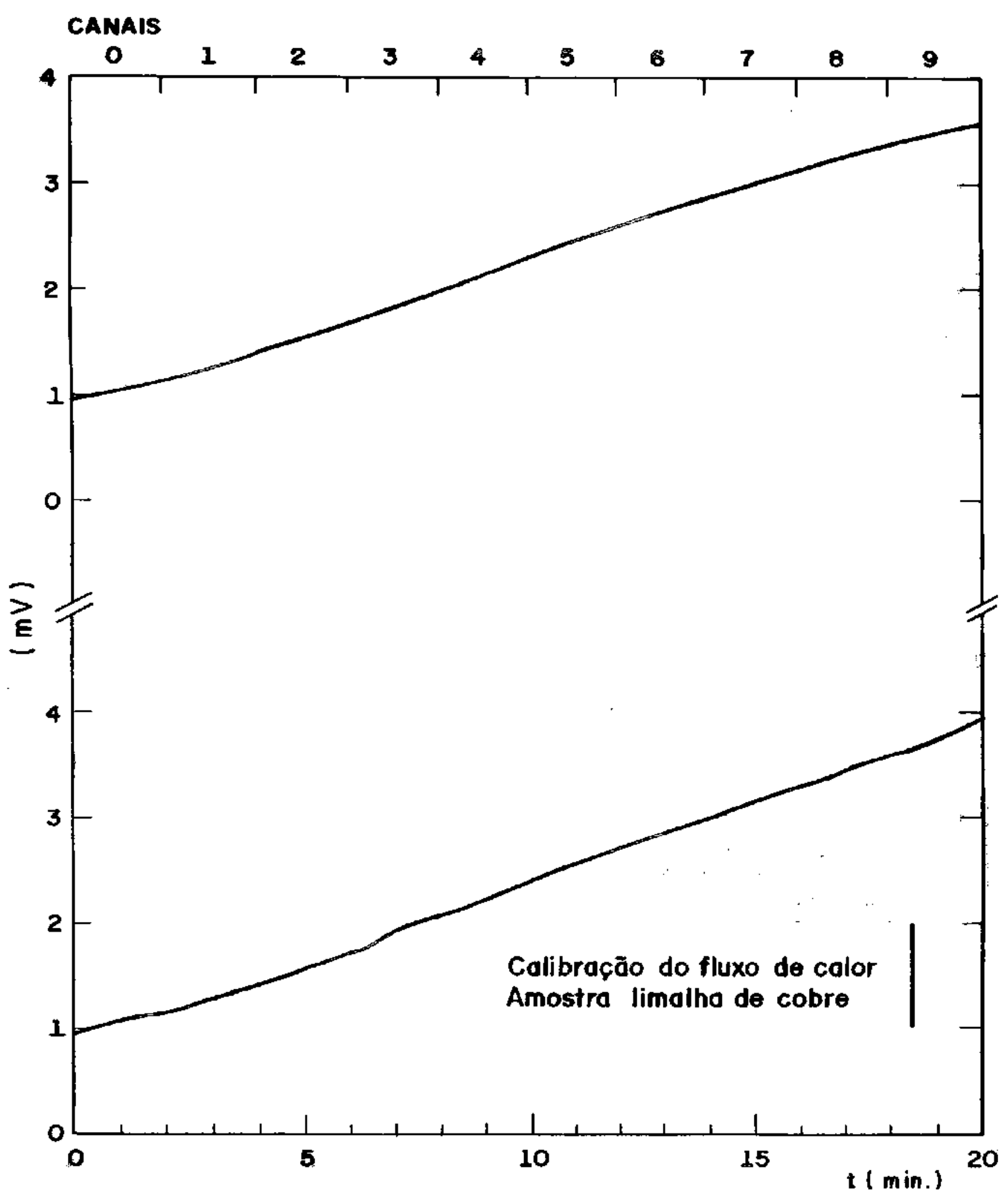

FIGURA 4 - Curva experimental do processo de aquecimento, a fluxo de calor constante, das amostras utilizando o método de controle por faixas de temperatura

A Figura 4 mostra a calibração do fluxo de calor, com o uso da técnica de aquecimento por canais. O interior do porta-amostras foi totalmente preenchido com limalhas de cobre compactadas. Tal material, pode ser idêntico ao da casca cilíndrica do porta-amostras onde está o termopar de referência, deve apresentar uma curva de aquecimento uniforme com o tempo, ou seja, uma reta. O aquecimento faz-se de modo controlado, para se conseguir em cada etapa, e no todo, um fluxo de calor constante. Consegue-se o aquecimento com taxa constante, por faixas de temperaturas, ajustando-se, na calibração, o tempo de funcionamento de cada canal. O intervalo total de temperaturas (ou intervalo total de tempo) é dividido em pequenos intervalos de temperaturas

Semina, Ci. Exatas/Tecnol., v. 13, n. 4, p. 231-237, dez. 1992 
(ou de tempo), a fim de tomarmos em cada intervalo, apenas o segmento da curva de Newton que se aproxima de uma reta. O limite da curva, para esta reta, é obtido expandindo-se em série a parte exponencial da equação (7)

$$
\begin{array}{r}
e^{-\alpha \cdot t}=1+(-\alpha \cdot t)+ \\
\frac{(-\alpha . t)^{2}}{2 !}+\frac{(-\alpha \cdot t)^{3}}{3 !}+\ldots
\end{array}
$$

onde, desprezando-se os termos não lineares, tem-se;

$$
T=T_{i}+\left(T_{f}-T_{i}\right) \cdot \alpha \cdot t
$$

que é a equação de uma reta. Desta forma, cada canal aquece a amostra linearmente.

\section{3 - CONCLUSÃO}

Discutimos a aplicabilidade da Aproximação do Corpo Uniforme, para sistemas porta-amostras de dimensões reduzidas, em Análise Térmica Diferencial, com base no aquecimento ou esfriamento Newtoniano, conforme a equação (7). Esta aproximação foi testada em vários experimentos (STORI, 1990; JURAITIS et al, 1989; MELLO, 1988), mostrando-se válida. Possibilitou a utilização de uma inovadora técnica de aquecimento ou esfriamento por canais (faixas crescentes de temperaturas quase-uniformes) idealizada por nosso grupo (STORI, 1990), a ser publicada.

O aquecimento ou esfriamento é feito em etapas consecutivas, de modo controlado, para se conseguir em cada etapa, e no todo, um fluxo de calor constante, o que, por sua vez, é conseguido ajustando-se, na calibração do fluxo, o tempo de aquecimento de cada canal.

Os resultados obtidos nos vários experimentos e a boa precisão da calibração mostrada na Figura 4 nos possibilitam concluir que a Aproximação de Corpo Uniforme constitui um método possível de ser usado em Análise Térmica Diferencial, quando o porta-amostras tem dimensões reduzidas.

DOMICIANO, J.B.; JURAITIS, K.R.; STORI, A. Uniform body approximation method for reduced dimensioning sample holders in differential thermal analysis. Semana: Ci. Exatas/Tecnol., Londrina, v. 13, n.4,

p. 231-237, Dez. 1992.

ABSTRACT: Many methods can be used to study the thermal properties of materials by the Differential Thermal Analysis (DTA) technique. The usual experimental method to get DTA curve demands the building of the sample holders with previously planned geometry to make the theoretical approach to the heat transmission easier. The description of this approach can be complex. In this article, the application of the Uniform Body Approximation Method, to reduced dimensioning sample holders in DTA, is described. One of the advantages of this method consists of considering the heating or the cooling of the sample and sample holders system as a Newtonian heating or cooling. This simplifies the theoretical approach.

KEY-WORDS: Differential Thermal Analysis; Heat Transfer

REFERÊNCIAS BIBLIOGRÁFICAS

BROWN, M.E. Introduction to thermal analysis: techniques and applications. London: Chapman and Hall, 1988.

JURAITIS, K.R.; DOMICIANO, J.B.; SANO, W.; DOTTO, C.T. Mátodo de andílise térmica diferencial através da técnica de fluxo de calor conhecido. Semina, Londrina, v. 10, n. 4, p. 267-271, 1989.

MELLO, C.B.M. de Análise témica e ressonancia paramagnética eletrónica de cloretos de niquel hidratados. Dissertaçăo (Mestrado) - Instituto de Fisica, Universidade de Seo Paulo, 1988.
SISSOM, L.E.; PITTS, D.R. Fenômenos de transporto. Rio de Janeiro: Guanabara, 1979.

STORí, A. Calibração de um analisador térmico diferencial construído no Laboratório de Materiais e Instrumentaçáo. Monografia (Especialização em Flsica) - Universidade Estadual de Londrina, 1990.

WENDLANDT, W.M.W. Thermal analysis. New York: John Wiley \& Sons, 1986.

Recebido para publicação em 10/2/1993 